\title{
A Rare Case of Mixed Ductal Neuroendocrine Tumor of the Pancreas
}

\author{
Vidya Viswanathan $^{1 *}$, Harsh Kumar ${ }^{1}$, Charusheela Gore ${ }^{1}$, Shrikant Kurhada ${ }^{2}$ and Rumaanah Khan ${ }^{1}$ \\ ${ }^{1}$ Department of Pathology, Dr D Y Patil Medical college Hospital and Research Centre \\ ${ }^{2}$ Consultant Laparoscopic Surgeon, Dr D Y Patil Medical college Hospital and Research Centre
}

\begin{abstract}
Collision tumors are tumors that have at least two types of tumors in the same anatomical site with no area of mixing within the transition zone. In 2010 WHO classification of neuroendocrine tumors consists of an adenocarcinoma component and a neuroendocrine carcinoma component in which each of the components accounts for $30 \%$ of the tumor. Such tumors are defined as mixed adenoneuroendocrine carcinomas. Occurrence of exocrine and endocrine tumors of the pancreas is extremely rare. The aim of our study was to describe a case in a 60 years old male who was diagnosed with this rare tumor. Gross, microscopic features and immunohistochemistry were used to diagnose this rare condition. Immunohistochemistry markers such as synaptophysin, chromogranin, EMA and Pan CK were used to come to a definitive diagnosis. Synaptophysin and chromogranin were found to be positive in the neuroendocrine component. EMA and Pan CK were found to be positive in the ductal component. Hence a diagnosis of mixed ductal neuroendocrine tumour (collision tumor) was made.
\end{abstract}

Keywords: Collision Tumor, Mixed, Rare, IHC

\section{Introduction}

Collision tumors are tumors that have at least two types of tumors in the same anatomical site with no area of mixing within the transition zone. ${ }^{[1]}$ In 2010 WHO classification of neuroendocrine tumors consists of an adenocarcinoma component and a neuroendocrine carcinoma component in which each of the components accounts for $30 \%$ of the tumor. Such tumors are defined as mixed adenoneuroendocrine carcinomas. ${ }^{[2]}$ Occurrence of exocrine and endocrine tumors of the pancreas is extremely rare. ${ }^{[2,3]}$ Pancreatic neuroendocrine tumors express markers such as synaptophysin, neuron specific enolase and chromogranin-A. ${ }^{[4]}$ Sometimes these tumors are associated with acute or chronic pancreatitis which may be due to obstruction of the pancreatic duct by the tumor. ${ }^{[5]}$ In this article we present a rare case of mixed adenoneuroendocrine pancreatic carcinoma

\section{Case Report}

A 60-year-old male patient came with chief complaints of pain abdomen more over the left hypochondrium and epigastric region since 6 months which was associated with weight loss of $10 \mathrm{kgs}$ in 3 months. He did not have any history of vomiting, loose stools, chest pain, palpitations, Breathlessness. He was a known Case of diabetes mellitus since 18 months and is on regular treatment for the same. There was history of repeated attacks of pancreatitis in the past. No History of hypertension, bronchial asthma, T.B or thyroid disorders was found. Family medical history was not contributory in this case .Personal history the patient consumed mixed diet, had normal sleep, decreased appetite, normal bowel and bladder habits. Past history of alcohol consumption present.

On examination pulse rate was 70/min, Blood Pressure was 130/90 mm Hg, No pallor, icterus or pedal edema. Systemic examination showed mild epigastric tenderness and no palpable mass. The hematological parameters were as follows Hb- $12.6 \mathrm{~g} / \mathrm{dl}, \mathrm{WBC}-5700$ cells/ cumm3, Platelet count - 1.9 lakhs, Blood group - A positive, Urea $37 \mathrm{mg} / \mathrm{dl}$, S. Creat - $1.1 \mathrm{mg} / \mathrm{dl}, \mathrm{Na}-138 \mathrm{mmol} / \mathrm{L}$, Potassium - $4.5 \mathrm{mmol} / \mathrm{dL}$, Fasting blood Glucose - $156 \mathrm{mg} / \mathrm{dL}$, T. Bilirubin - $0.7 \mathrm{mg} / \mathrm{dL}$, SGOT - $38 \mathrm{IU}$, SGPT - $30 \mathrm{IU}$, ALP - $92 \mathrm{IU} / \mathrm{L}$, Protein - $5.6 \mathrm{~g} / \mathrm{dL}$, Albumin - $3.9 \mathrm{~g} / \mathrm{dL}$, Serum amylase - 900 U/L, Serum lipase - 1600 U/L, HIV / HbsAg - negative. Only fasting blood glucose and serum amylase levels were found to be raised all other parameters were within normal limits. Chest X Ray showed normal radiographic study. ECG showed normal sinus rhythm. Echo showed ejection fraction of $60 \%$, no regional wall motion abnormalities were noted. Mild concentric LVH was seen.

Endoscopic ultrasound guided fine needle aspiration cytology from pancreatic head mass showed only a few atypical cells which was reported as suspicious of malignancy.

CT of abdomen and pelvis showed severe atrophy of pancreatic parenchyma in the region of uncinate process, 
head, body and tail with dilatation of main pancreatic duct. Poorly enhancing soft tissue in the region of the neck and adjacent head of pancreatic parenchyma measuring 2 $\mathrm{X} 2.5 \mathrm{X} 1.7 \mathrm{cms}$ in maximum diameter. The CT findings suggested the possibility of pancreatic adenocarcinoma.

Ultrasound of the abdomen and pelvis showed fatty liver along with borderline prostate enlargement.

Subsequent to this the patient was posted for Whipple's procedure that is Pancreatico-duodenectomy along with cholecystectomy and feeding jejunostomy under general anaesthesia. Post-surgery the specimen was sent to Dept of Pathology for Histopathological examination.

In our department the specimen received was studies for both gross and microscopic features.

On gross examination the external surface of the pancreas was greyish brown in colour. Cut surface was_ill defined, yellowish-white in colour, firm in consistency. Very scant normal pancreatic parenchyma was seen (figure 1\&2)

Microscopic examination showed a well differentiated tumor predominantly composed of ductal invasive component ( figure 3\&4 ) with approximately one third of neuroendocrine component ( figure 5\&6). The tumor was seen to extend into peripancreatic fat and the adjoining duodenum (figure 7). There was no evidence of Lymphovascular invasion but perineural invasion was present (figure 9\&10). The adjoining pancreas showed features of chronic pancreatitis with fibrosis (figure 8). All the surgical margins were found to be free of tumor. Four lymph nodes were identified one of them was seen to be involved by the tumor. One of the lymph nodes also showed

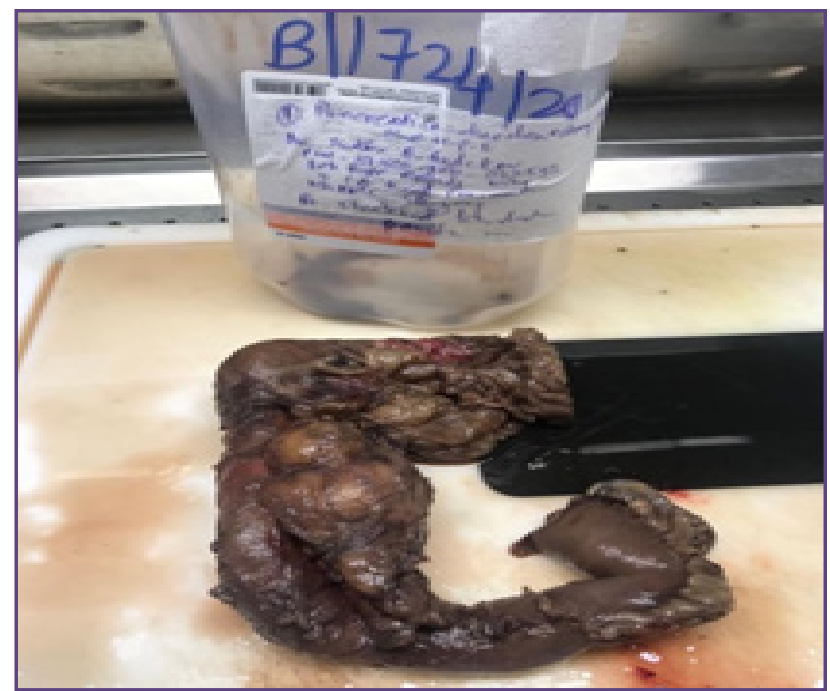

Fig.1: Grosspictureoftheentirepancreaticoduodenectomy specimen. features of non-caseating granuloma. A diagnosis of mixed ductal neuroendocrine carcinoma was made based on the above microscopic findings. Immunohistochemistry was performed to confirm the diagnosis. Synaptophysin and chromogranin (figure 11\&12) were found to be positive in the neuroendocrine component. Epithelial membrane antigen and pan CK (figure 13\&14) were found to be positive in the ductal component.

\section{Discussion}

We report an exceeding rare case of pancreatic adenocarcinoma and neuroendocrine tumor. According to WHO classification collision tumors are composed of two different malignancies without histological admixture. ${ }^{[1,3]}$ Another terminology MANEC that is mixed adenoneuroendocrine carcinoma was introduced by WHO in 2010. Tumors with two different malignant components with each tumor accounting for $30 \%$ of the tumor are classified as MANEC. ${ }^{[2,7]}$ Collision tumors have been reported in various organs of the body such as breast, uterus, colon and stomach. They most commonly occur in the stomach and oesophagus. ${ }^{[1]}$

The pancreas is composed of both exocrine and endocrine components. Pancreatic collision tumors are rare with limited literature. Peak incidence of these tumors is between 30-60yrs. ${ }^{[5]}$ Diagnosis of pancreatic collision tumors is a post-operative diagnosis based on histopathological examination. ${ }^{[1]}$ Collision tumors appear to have a poor prognosis with a median survival of 10 months. Surgical treatment appears to be the treatment of choice. ${ }^{[1,2,4,5]}$

Little is known about the carcinogenesis of these tumors. One hypothesis states that there may be dysfunction of

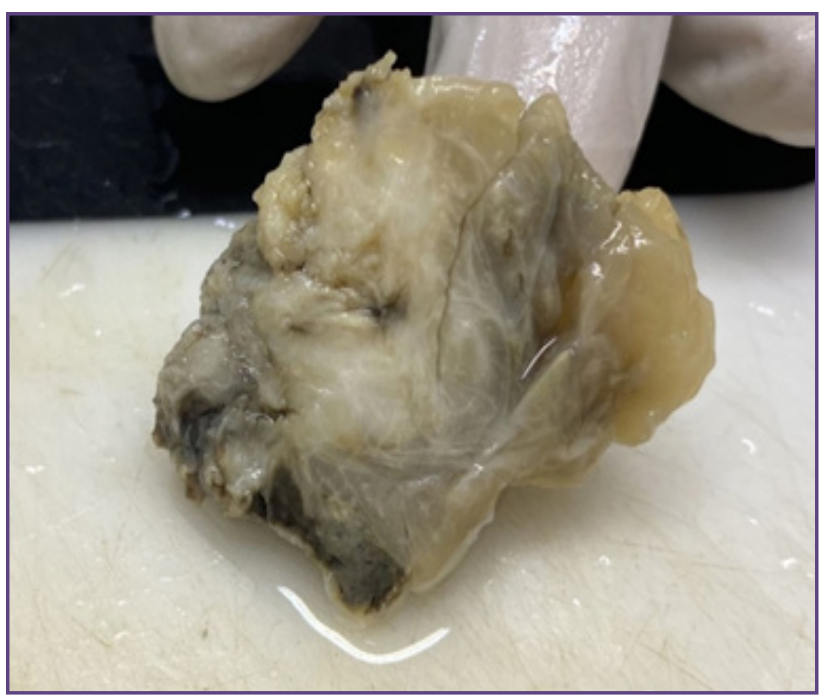

Fig. 2: Gross picture of the tumor proper. 


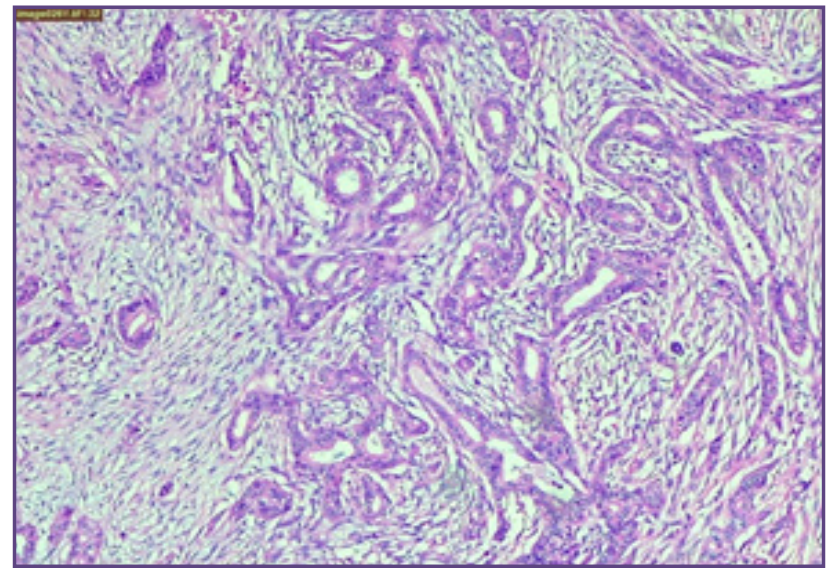

Fig. 3: Low power view(10X) in Hematoxylin and eosin stain of Infiltrating, haphazard glands and surrounding desmoplastic stroma.

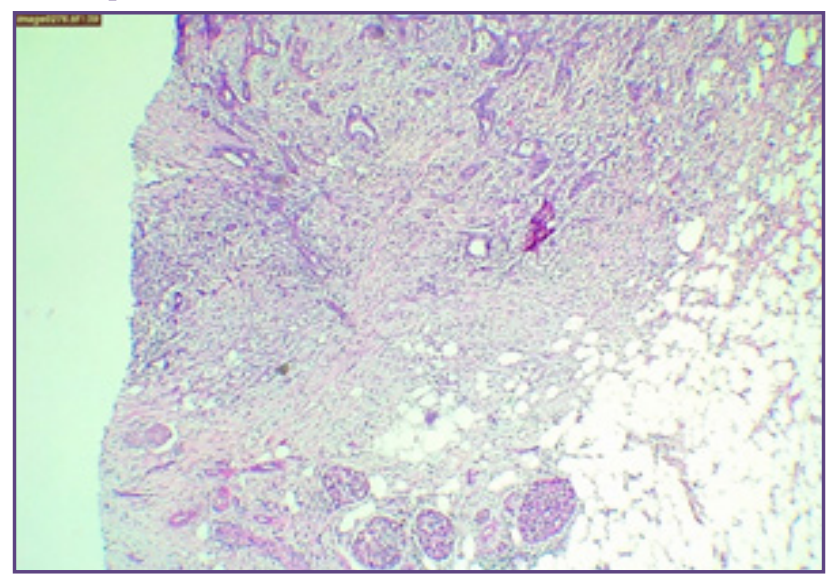

Fig. 5: Scanner view(4X) in Hematoxylin and eosin stain of an area demonstrating components of neuroendocrine tumor (lower-most) and pancreatic ductal adenocarcinoma (upper)

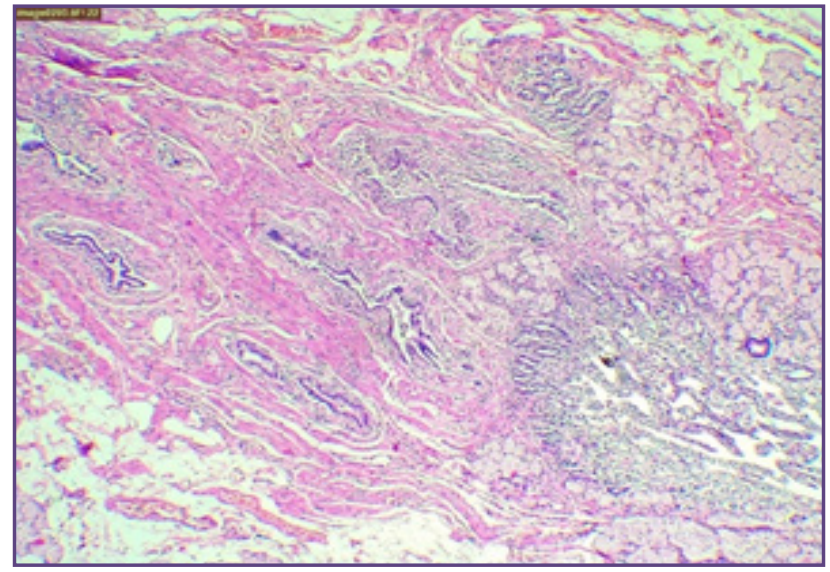

Fig. 7: Low power view(10X) in Hematoxylin and eosin stain of infiltration into duodenal wall

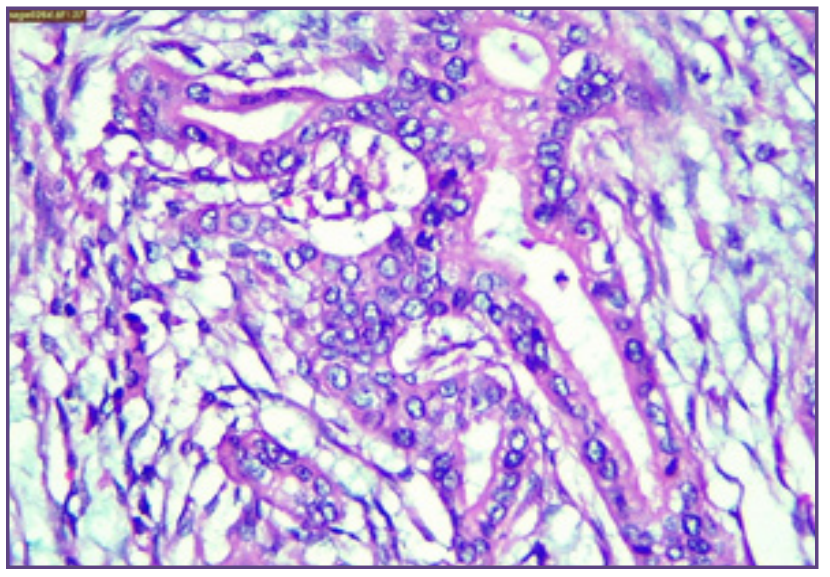

Fig. 4: High power view(40X) in Hematoxylin and eosin stain showing multilayering,nuclear atypia, Loss of polarity and focal prominent nucleoli.

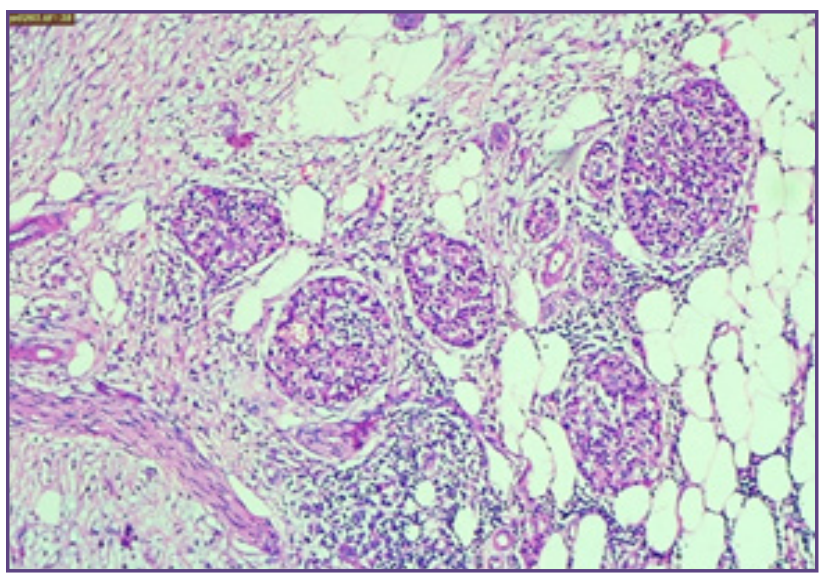

Fig. 6: Scanner view(4X) in Hematoxylin and eosin stain of an area demonstrating components of neuroendocrine tumor (lower-most) and pancreatic ductal adenocarcinoma (upper).

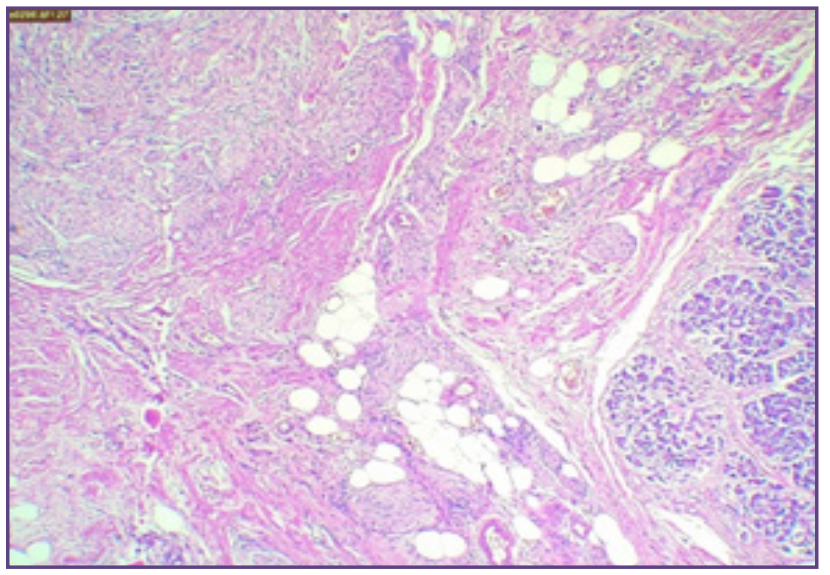

Fig. 8: Low power view(10X) in Hematoxylin and eosin stain of chronic pancreatitis on background showing fibrosis, inflammation and fatty infiltration. 


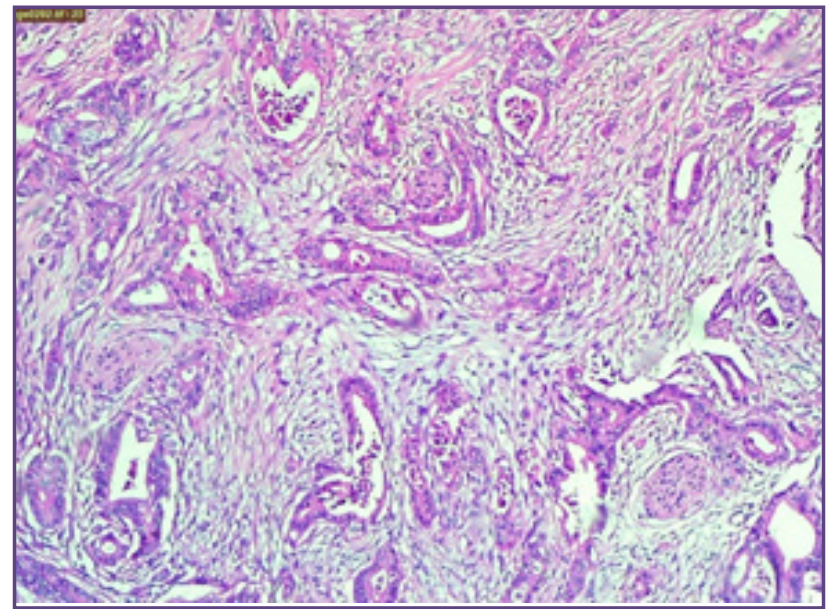

Fig. 9: Low power view(10X) in Hematoxylin and eosin stain of perineural invasion.

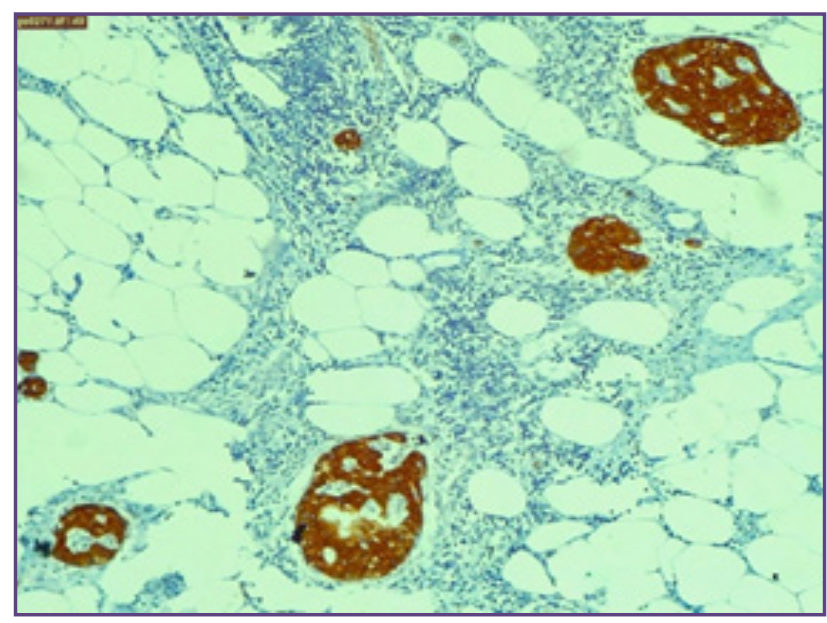

Fig. 11: Synaptophysin positivity in the neuroendocrine component.

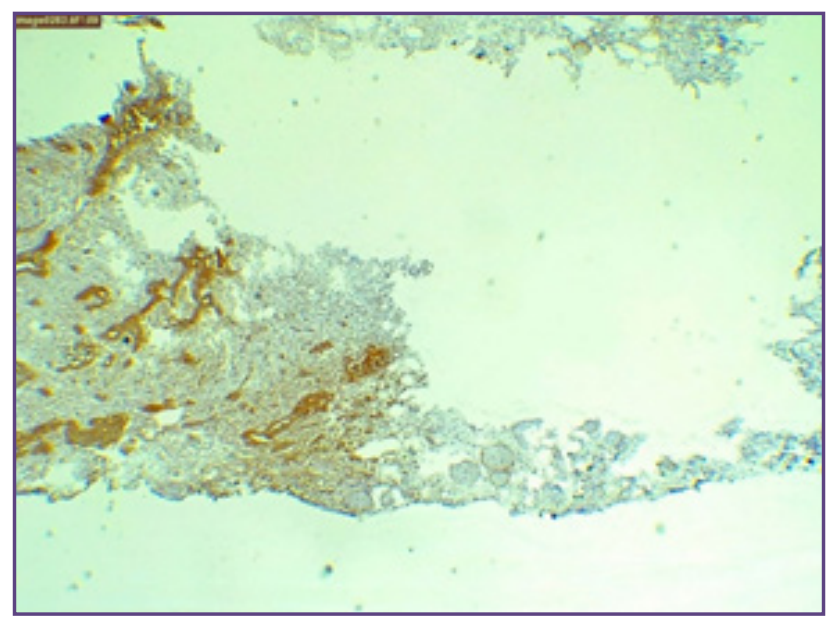

Fig. 13: EMA positivity in the Glandular component.

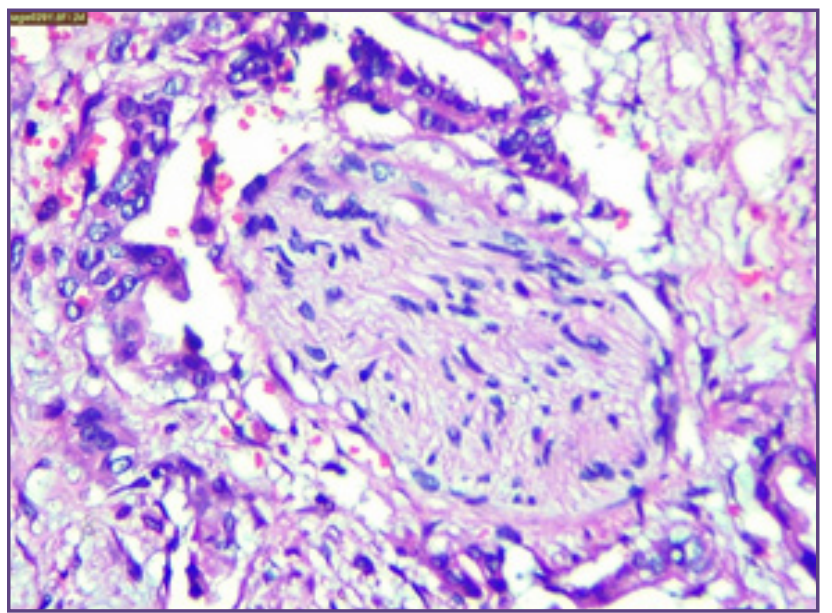

Fig. 10: High power view(40X) in Hematoxylin and eosin stain of perineural invasion.

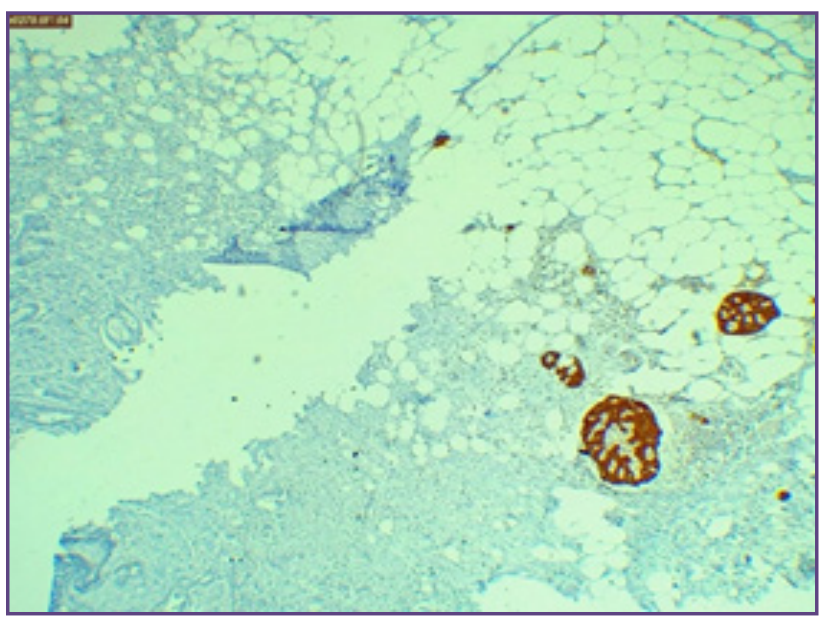

Fig. 12: Chromogranin positivity in neuroendocrine component.

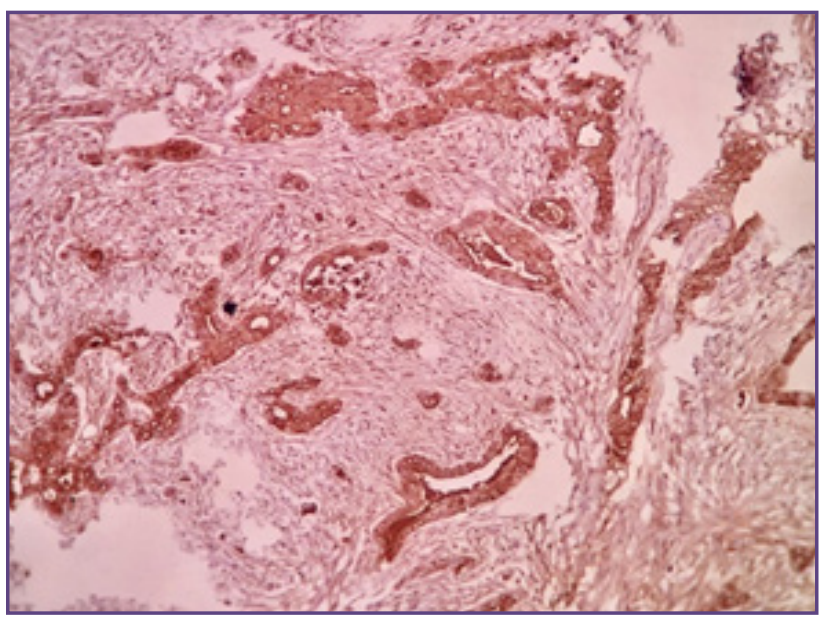

Fig. 14: PAN CK positivity seen in adenocarcinoma. 
multiple tumor suppressor gene that causes inadequate repair of genes. ${ }^{[1]}$ Mixed ductal endocrine tumors of the pancreas have a common endodermal histogenesis for the ductal and endocrine cells in the pancreas. ${ }^{[6]}$

Fitzgerald reported mixed type tumors as $0.2 \%$ of all tumors. ${ }^{2]}$ These tumors have been designated the term mixed ductal acinar tumors etc based on their components. Chang et al classified mixed tumors as amphicrine type, mixed type, collision type, solitary concomitant type and multiple concomitant type. ${ }^{[7]}$

Pancreatic neuroendocrine tumors can be functional and non-functional tumors. Functional tumors release active hormones in peripheral blood which are responsible for a particular syndrome. Non-functional tumors secrete functionally inert hormones which do not produce any syndrome. ${ }^{[5]}$ Histopathologically non-functional tumors cannot be distinguished from functional tumors. However positive staining for chromogranin and synaptophysin confirms the diagnosis of non-functional pancreatic neuroendocrine tumors. ${ }^{[4,5]}$

Immunohistochemistry is very important for the diagnosis of pancreatic neuroendocrine tumors. Several markers are available such as neuron specific enolase, CD56, synaptophysin and other hormones. The malignant or metastatic potential of the neuroendocrine tumor can be determined by tumor size, expression of Ki67, vascular invasion and perineural invasion. ${ }^{[5,6]}$ Most of these tumors are also associated with pancreatitis. In our study the association of chronic pancreatitis and neuroendocrine tumor seem incidental. ${ }^{[5]}$

In this article we report a case of mixed adenoneuroendocrine tumor or collision tumor. The exact method of tumorigenesis is unknown. When faced with a pancreatic mass with atypical radiological features and also ill-defined mass on gross examination a diagnosis of collision tumor needs to be considered. ${ }^{[1]}$ The diagnosis can be confirmed by using additional immunohistochemical methods.

\section{Conclusion}

Pancreatic mixed adenoneuroendocrine tumors are extremely rare which raise important diagnostic and therapeutic decisions. Association with chronic pancreatitis is also noted which is not fully understood. Histopathology and immunohistochemistry remain the gold standard for the diagnosis of these tumors.

\section{References}

1. Wang Y, Gandhi S, Basu A, Ijeli A, Kovarik P, Sekosan M, Demetria M. Pancreatic Collision Tumor of Ductal Adenocarcinoma and Neuroendocrine Tumor. ACG Case Rep J. 2018 May 23;5:e39.

2. Kouki Imaoka, Saburo Fukuda, Hirofumi Tazawa, Yoshio Kuga, Tetsuya Mochizuki, Yuzo Hirata, Seiji Fujisaki, Mamoru Takahashi, Toshihiro Nishida, Hideto Sakimoto. A mixed adenoneuroendocrine carcinoma of the pancreas: a case report. Surgical case reports (2016)2:133

3. Serafini S, Da Dalt G, Pozza G, et al. Collision of ductal adenocarcinoma and neuroendocrine tumor of the pancreas: a case report and review of the literature. World J Surg Oncol. 2017;15(1):93. Published 2017 May 2. doi:10.1186/ s12957-017-1157-9

4. Amador Cano, A., García, F., Espinoza, A., Bezies, N., Herrera, E., \& De Leija Portilla, J. (2013). Nonfunctional neuroendocrine tumor of the pancreas: Case report and review of the literature. International journal of surgery case reports, 4(2), 225-228.

5. Surlin, V., Ramboiu, S., Ghilusi, M. et al. Incidental intraoperative discovery of a pancreatic neuroendocrine tumor associated with chronic pancreatitis. Diagn Pathol 7, 132 (2012).

6. Leteurtre Emmanuelle, Brami F, Kerr-Conte J, Quandalle $\mathrm{P}$, Lecomte-Houcke M.mixed ductal endocrine carcinoma of the pancreas. A case study with mixed ductal endocrine metastasis double labelled for cytokeratin19 and synaptophysin. Arch Pathol Lab Med 2000, Vol 124; 284286.

7. Shimada N, Miwa S, Arai T, Kitagawa N, Akita S, Linuma $\mathrm{N}$, Ishii K. cystic mixed adenoneuroendocrine carcinoma of the pancreas: A case report.international journal of surgery case reports. Vol 52;2018; Pg 1-4.

*Corresponding author:

Dr Vidya Viswanathan, Dr D Y Patil medical college hospital and research centre, Dr D Y Patil Vidyapeeth, Pune

Phone: +91 9822046668

Email: docvidya11@gmail.com

Financial or other Competing Interests: None.

Date of Submission $\quad: 13 / 10 / 2020$

Date of Final Revision : 13/02/2021

Date of Acceptance $\quad: 15 / 02 / 2021$

Date of Publication $\quad: 28 / 02 / 2021$ 